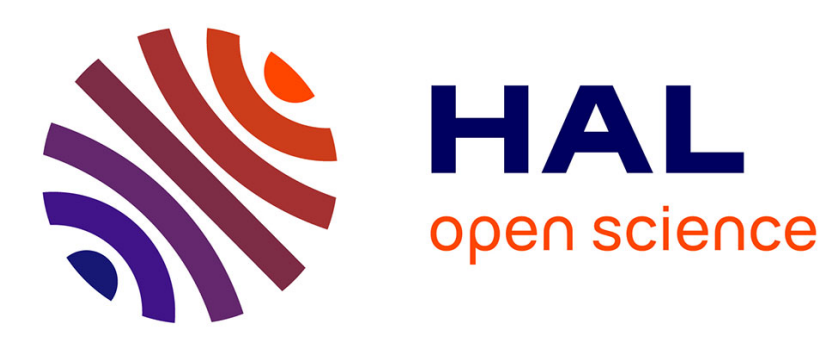

\title{
Ultrasonic characterization of porous silicon using a genetic algorithm to solve the inverse problem
}

\author{
Julien Bustillo, Jérôme Fortineau, Gaël Gautier, Marc Lethiecq
}

\section{To cite this version:}

Julien Bustillo, Jérôme Fortineau, Gaël Gautier, Marc Lethiecq. Ultrasonic characterization of porous silicon using a genetic algorithm to solve the inverse problem. NDT International, 2013, 62, pp.93-98. 10.1016/j.ndteint.2013.11.007 . hal-01080736

\section{HAL Id: hal-01080736 \\ https://hal.science/hal-01080736}

Submitted on 6 Nov 2014

HAL is a multi-disciplinary open access archive for the deposit and dissemination of scientific research documents, whether they are published or not. The documents may come from teaching and research institutions in France or abroad, or from public or private research centers.
L'archive ouverte pluridisciplinaire HAL, est destinée au dépôt et à la diffusion de documents scientifiques de niveau recherche, publiés ou non, émanant des établissements d'enseignement et de recherche français ou étrangers, des laboratoires publics ou privés. 


\title{
Ultrasonic characterization of porous silicon using a genetic algorithm to solve the inverse problem
}

\author{
J. Bustilloa,b,*, J. Fortineau ${ }^{\mathrm{b}, \mathrm{a}}$, G. Gautier ${ }^{\mathrm{a}}$, M. Lethiecq ${ }^{\mathrm{a}, \mathrm{b}}$ \\ ${ }^{a}$ GREMAN, University of Tours, Parc de Grandmont, 37200 Tours, France \\ ${ }^{b}$ GREMAN, ENI Val de Loire, 3 rue de la chocolaterie, 41000 Blois, France
}

\begin{abstract}
This paper presents a method for ultrasonic characterization of porous silicon in which a genetic algorithm based optimization is used to solve the inverse problem. A one dimensional model describing wave propagation through a water immersed sample is used in order to compute transmission spectra. Then, a water immersion wide bandwidth measurement is performed using insertion/substitution method and the spectrum of signals transmitted through the sample is calculated using Fast Fourier Transform. In order to obtain parameters such as thickness, longitudinal wave velocity or density, a genetic algorithm based optimization is used.

A validation of the method is performed using aluminum plates with two different thicknesses as references: a good agreement on acoustical parameters can be observed, even in the case where ultrasonic signals overlap.

Finally, two samples, i.e. a bulk silicon wafer and a porous silicon layer etched on silicon wafer, are evaluated. A good agreement between retrieved values and theoretical ones is observed. Hypothesis to explain slight discrepancies are proposed.
\end{abstract}

Keywords:

genetic algorithm, inverse problem resolution, ultrasonic NDE, porous silicon

\section{Introduction}

Analysis of ultrasonic waves which have been transmitted through a sample allow acoustic and hence mechanical parameters of the sample to be extracted. In most cases, signals are not overlapped and both time domain [1] and frequency domain $[2,3]$ analysis can be used to determine parameters such as wave velocity, attenuation or density.

In some cases, the acoustic wave transmission coefficients in frequency domain have been used in order to calculate these parameters $[4,5]$.

When thickness of samples are in the same order as the wavelenght in the medium, or in case of multilayer samples, overlapping can occur and direct measurements of parameters is not longer possible.

Nevertheless, ultrasonic non-destructive characterization of materials has been widely studied in the case of thin layers $[6,5,7,8]$. Given the complexity of received signals, modelbased methods are proposed [8]. Using an inverse problem resolution, these parameters can be extracted [9]. However, most optimization methods need a guess of initial values [10]. In the case of material whose parameters have huge variations, it is difficult to guess initial values with sufficient accuracy to reach the correct solution.

In this study, a genetic algorithm based resolution is purposed to limit the impact of initial values. Indeed, this optimization

\footnotetext{
*tel:+33254558440

Email address: julien.bustillo@univ-tours.fr (J. Bustillo)
}

method is known to converge towards the global solution [11] and to ensure unicity of solution. A 1-D wave propagation model is chosen to calculate the spectrum of the signal transmitted through multilayer sample. This spectrum is dependent on geometrical and acoustical properties of each layer, such as thickness, wave velocity and density.

For validation purposes, the theoretical transmission spectrum of immersed aluminum plates is calculated and compared to experimental ones in order to retrieve acoustical parameters of the sample.

Then, a sample composed of a porous silicon ( $\mathrm{PoSi}$ ) layer etched on a silicon wafer is studied. Wave velocity and density of bulk silicon are known. The porous silicon layer is considered as homogeneous and its parameters are estimated by solving the inverse problem with genetic algorithm.

\section{Porous silicon}

\subsection{Fabrication of the Porous Silicon layers}

Porous Silicon has found many applications in microelectronics. One can point out for example, the use of mesoporous $\mathrm{Si}$ as an isolating substrate for RF applications [12] or the application of the high specific surface of PoSi in sensors[13].

The PoSi layers were formed by the anodization in HF based solutions of highly doped (100) p-type $\mathrm{Si}(10-50 \mathrm{~m} \Omega$.cm) samples with thicknesses varying between 650 and $700 \mu \mathrm{m}$. This type of silicon is known to produce mesoporous materials with pore diameters between 10 and $100 \mathrm{~nm}$ [14]. Etching process is 
highly anisotropic and pores direction is normal to the wafer surface as can be seen in figure 1 . The electrochemical etching was performed in a double tank electrochemical cell developed by AMMT. The HF concentration is $30 \%$ and the surfactant used is acetic acid with volume ratios HF (50\%): Acetic acid: $\mathrm{H}_{2} \mathrm{O}$ of $4.6: 2.1: 1.5$. The anodization was performed in a galvanostatic mode. A current density of $28 \mathrm{~mA} / \mathrm{cm}^{2}$ was fixed to obtain an estimated porosity of $50 \%$. Then, the duration determined the total thickness of the porous layers. In our case, a duration of 174 minutes lead to $200 \mu \mathrm{m}$. The average technologica dispersion is in the range of 5 to $10 \%$. Decontamination of samples is performed using several baths of pure water so that etching liquid is completely removed

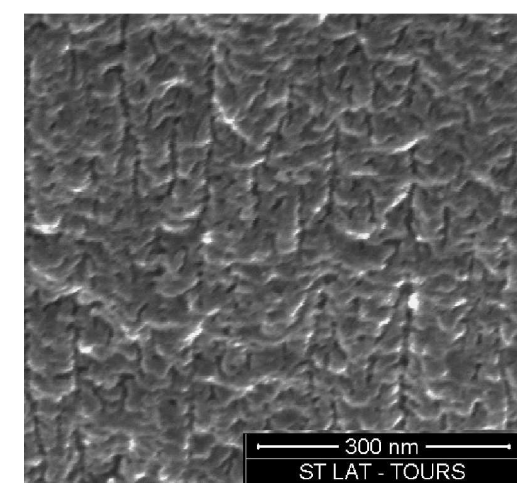

Figure 1: SEM observations of a typical highly doped n-type mesoporous sample [12].

\subsection{Materials specification}

The samples used for this study are square-shaped crystalline silicon wafers on which a circular shaped PoSi layer with a one inch $(2.54 \mathrm{~cm})$ diameter is etched. This diameter is larger than the surface of the acoustic beam to ensure that all the ultrasonic signal passes through the porous medium. Porous silicon layer thickness has been measured using a destructive method in order to be compared with values retrieved using inverse problem resolution. These values and the expected density are recalled in table 1 .

Table 1: Sample geometrical characteristics.

\begin{tabular}{|c|c|c|c|}
\hline $\begin{array}{c}\text { Sample } \\
\text { number }\end{array}$ & $\begin{array}{c}\text { Measured wafer } \\
\text { thickness }\end{array}$ & $\begin{array}{c}\text { Measured } \\
\text { PoSi thickness }\end{array}$ & $\begin{array}{c}\text { Expected } \\
\text { PoSi density }\end{array}$ \\
\hline 1 & $674 \pm 1 \mu \mathrm{m}$ & $0 \mu \mathrm{m}$ & $/$ \\
\hline 2 & $675 \pm 1 \mu \mathrm{m}$ & $195-204 \mu \mathrm{m}$ & $1650 \pm 100 \mathrm{~kg} \cdot \mathrm{m}^{-3}$ \\
\hline
\end{tabular}

The physical parameters of the crystalline silicon and water used for this study are noted in Table $2[15,16]$. The ultrasonic wave velocity in water is strongly dependant on temperature [15]. In our study, the temperature of the water is kept around $20^{\circ} \mathrm{C}$ and is measured, allowing wave velocity in water to be corrected in calculations.

Porous silicon parameters are unknown and are the model inputs for optimization.
Table 2: acoustical parameters of porous silicon, silicon and water.

\begin{tabular}{|c|c|c|c|}
\hline $\begin{array}{c}\text { acoustical } \\
\text { parameter @ 20 }\end{array}$ & Water & Silicon & $\begin{array}{c}\text { porous silicon } \\
\text { variation range }\end{array}$ \\
\hline wave velocity $(\mathrm{m} / \mathrm{s})$ & 1480 & 8430 & $1480-8430$ \\
\hline density $\rho\left(\mathrm{kg} / \mathrm{m}^{-3}\right)$ & 1000 & 2330 & $1000-2330$ \\
\hline
\end{tabular}

\section{Inverse problem resolution}

\subsection{Model}

The acoustic wave propagation model is based on the assumption of a 1-D plane wave propagation along the $\mathrm{z}$ axis where the ultrasonic beam is normal to the surface and all the interfaces are parallel. Given that wavelengths in the considered bandwidth are more than 3 orders of magnitude larger than the pore size, the porous silicon layer is considered as a non-dispersive medium. In this study, all the measurements are performed using a water immersion method. Given the small deformation hypothesis, the acoustic velocity $v$ in any one of the layers can be decomposed into a scalar and a vector potential field, respectively $\phi$ and $\psi$ :

$$
u=\nabla \phi+\nabla \wedge \psi
$$

with

$$
\begin{aligned}
& \phi=\left[a_{+}^{L} e^{j k_{z}^{L} z}+a_{-}^{L} e^{-j k_{z}^{L} z}\right] e^{-j \omega t} \\
& \psi=\left[a_{+}^{S} e^{j k_{z}^{S} z}+a_{-}^{S} e^{-j k_{z}^{S} z}\right] e^{-j \omega t}
\end{aligned}
$$

where $a_{-}^{U}$ is the downstream amplitude, $a_{+}^{U}$ the upstream amplitude and $k_{z}^{U}$ the complex wavevector (eq. 3 ) of mode $\mathrm{U}$, which can either be shear (S) or longitudinal (L), and $\omega$ is the angular frequency.

$$
\begin{aligned}
& \left|k_{z}^{L}\right|=\frac{\omega}{c_{z}^{L}} \\
& \left|k_{z}^{S}\right|=\frac{\omega}{c_{z}^{S}}
\end{aligned}
$$

where $c_{z}^{U}$ is wave velocity of mode $\mathrm{U}$.

The matrix representation proposed by Cervenka [17] is used in this study. Each of the layers of the material can be either fluid or solid. In particular, the pseudo-fluid matrix proposed in his work allows the entire system to be represented by a single matrix. Since the sample is surrounded by water and the waves are normal to its surfaces, shear waves can be neglected, hence vector potential field $\psi$ is null and displacement $u$ can be written as:

$$
u=\nabla \phi
$$

Using the material characteristics of each layer (longitudinal wave velocity, attenuation, density, thickness), the propagation of ultrasonic waves can be computed. Taking into account the boundary conditions, waves in the output medium $\left(A_{t}\right.$ transmitted 
through the sample) and waves in the input medium $\left(A_{i}\right.$ the incident wave and $A_{r}$ the wave reflected by the sample) are related through matrix $\mathscr{G}$ :

$$
\begin{gathered}
\text { input medium : }\left\{\begin{array}{l}
A_{i}=a_{+i n}^{L} e^{j k_{\text {zin }}^{L} z} e^{-j \omega t} \\
A_{r}=a_{-i n}^{L} e^{j k_{z \text { in }}^{L} z} e^{-j \omega t}
\end{array}\right. \\
\text { output medium : }\left\{\begin{array}{l}
A_{t}=a_{+ \text {out }}^{L} e^{j k_{\text {out }}^{L} z} e^{-j \omega t}
\end{array}\right.
\end{gathered}
$$

and

$$
\left[\begin{array}{c}
A_{t} \\
0
\end{array}\right]=\mathscr{G}\left[\begin{array}{c}
A_{i} \\
A_{r}
\end{array}\right]
$$

Eq. 6 can be rewritten to relate the two waves which can be measured,i.e. the transmitted wace $A_{t}$ and the reflected wave $A_{r}$, to the incident wave $A_{i}$.

$$
\left[\begin{array}{l}
A_{t} \\
A_{r}
\end{array}\right]=\left[\begin{array}{l}
\mathscr{M}_{11} \\
\mathscr{M}_{21}
\end{array}\right]\left[A_{i}\right]
$$

Using this notation, transmission and reflection coefficients can be computed:

$$
\begin{aligned}
& R=\frac{A_{r}}{A_{i}}=\mathscr{M}_{21}=-\frac{\mathscr{G}_{21}}{\mathscr{G}_{22}} \\
& T=\frac{A_{t}}{A_{i}}=\mathscr{M}_{11}=\frac{\mathscr{G}_{11} \mathscr{G}_{22}-\mathscr{G}_{21} \mathscr{G}_{12}}{\mathscr{G}_{22}}
\end{aligned}
$$

\subsection{Experimental setup}

In this study, an insertion-substitution method is used. Two measurements of transmitted waves are performed: one in the immersion medium and an other after sample insertion. Thus, attenuation in water, diffraction effects and both transducer transfer functions are corrected [18].

Received reference signal $r e f(t)$ is composed of an impulse response $h(t, f)$, an attenuation coefficient $\alpha_{w}$ and a diffraction effect term $D\left(d_{w}, f\right)$, where $d_{w}$ is the distance between both transducers.

$$
r e f(t)=h(t, f) \cdot e^{-\alpha_{w} \cdot d_{w}} \cdot D\left(d_{w}, f\right)
$$

Signal $s(t)$ considering the sample insertion is given by:

$$
s(t)=\beta_{0} \cdot r e f\left(t-\tau_{0}\right)+\sum_{i=1}^{\infty} \beta_{i} \cdot r e f\left(t-\tau_{i}\right)
$$

where parameters $\beta_{i}$ and $\tau_{i}$ are respectively amplitudes and delays of $i^{\text {th }}$ incoming wave. They depend on material acoustical properties.

A corrected transmission coefficient $T$ is defined as:

$$
T(f)=\frac{S(f)}{\operatorname{Ref}(f)}
$$

where $\operatorname{Re} f(f)$ and $S(f)$ are spectra of respectively $r e f(t)$ and $s(t)$.

The experimental setup is shown in Figure 2.

Two similar transducers are set facing each other, spaced by $8 \mathrm{~cm}$. Sample is placed at $4 \mathrm{~cm}$ of the emitter. The electrical excitation is delivered to the emitter using an Agilent 33250A waveform generator. Received signal is recorded by a LeCroy waveRunner 64XI digital oscilloscope at a sampling rate of $500 \mathrm{MS} / \mathrm{s}$. In order to increase signal to noise ratio, a 1024 sweep averaging is performed. No windowing has been applied to the received signal. However zero-padding process has been performed in order to increase the number of points of the spectra.

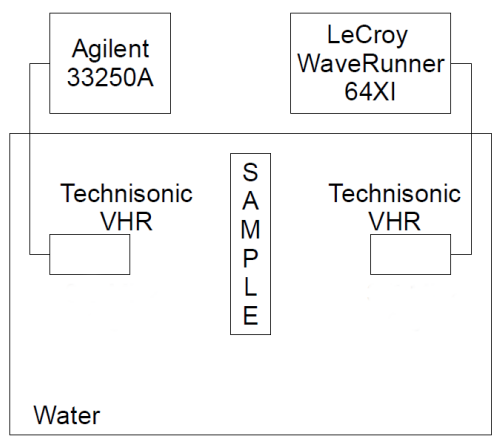

Figure 2: Insertion-substitution measurement: experimental setup.

For validation, $3.5 \mathrm{MHz}$ centre frequency transducers ISL0303VHR from Technisonic are used. They have 0.375 inch diameter active surfaces. The excitation voltage is a $10 \mathrm{~V}$ square pulse of $30 \mathrm{~ns}$ duration. For PoSi measurements, $20 \mathrm{MHz}$ centre frequency transducers ISL2002VHR from Technisonic with quarter inch diameter active surfaces are used. The excitation voltage is then a $10 \mathrm{~V}$ square pulse of $20 \mathrm{~ns}$ duration. Their reference signals are shown in figure 3.
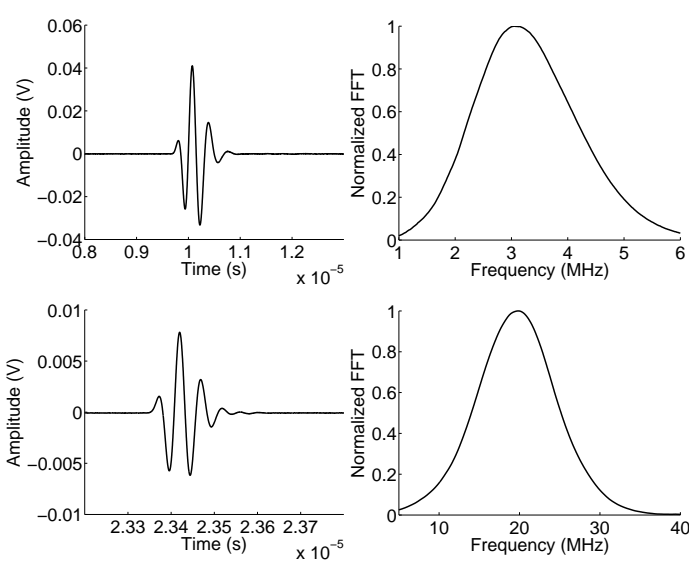

Figure 3: Temporal and spectral representation of reference signals for validation in aluminium(top) and measurements in PoSi etched wafers (bottom).

\subsection{Fitting strategy}

The optimization method used in this study is based on a Genetic Algorithm (GA). It was developed by Holland [19] and mimics a on natural selection process. Even if it was first designed for combinatorial problems, researchers have validated GA for optimization problems [20].

Most of the GA have been implemented in a binary representation because of its low cardinality [21] and its similarity with biological 
models. However some researchers have proposed to use a representation closer to systems. Following that, Goldberg has developed a floating point based genetic algorithm, better adapted to numerical optimization [22].

Convergence of GA is unique if its parameters (selection pressure, mutation, crossing over, population size) are well set, even if variable bounds are very large. Yet a drawback of GA is that its solution for a given number of generations is only an approximation of the global optimum. In order to converge at a closer value, Michalewicz proposed a non uniform mutation operator [23]. Input parameters can be refined during optimization, using an ageing effect. Eq.(12) and (13) describe a non uniform mutation operator, and more precisely the parameter $b$ defines nonlinear behavior.

$$
\begin{gathered}
g^{(k+1)}=\left\{\begin{array}{l}
g^{(k)}+\Delta\left(t, g_{\max }-g^{(k)}\right) \\
g^{(k)}-\Delta\left(t, g^{(k)}-g_{\text {min }}\right)
\end{array}\right. \\
\Delta(t, y)=y \cdot\left(1-r^{\left(1-\frac{t}{T}\right)^{b}}\right), r \in[0,1]
\end{gathered}
$$

A higher non-linearity of mutation will lead to a better final tuning of input parameters.

The selection process is performed using universal stochastic method [24]. Elistism, which consists in keeping the best individual is used during this study in order to improve the convergence of the GA [11].

Figure 4 shows how wave velocity and density can be extracted. Note that the theoretical spectrum calculation takes into account the measured reference spectrum $\operatorname{Re} f(f)$.

The fitness calculation is based on the root mean square distance between theoretical and experimental curves in Fourier domain.

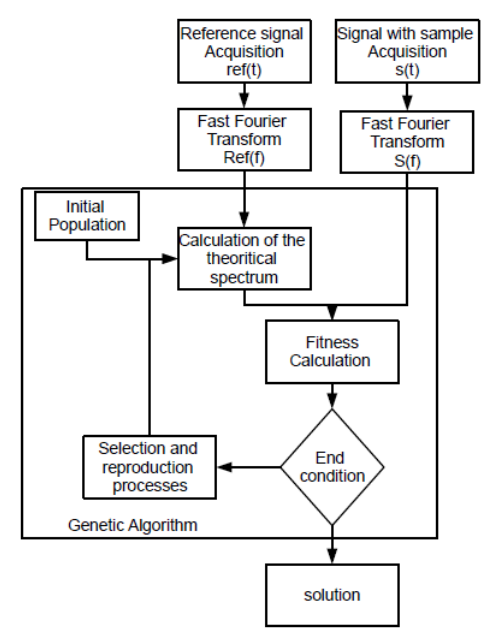

Figure 4: Data processing scheme.

\section{Validation}

A validation is performed using two aluminum plates with different thicknesses : $4 \mathrm{~mm}$ and $1.45 \mathrm{~mm}$ (respectively sample 1 and 2). This material is chosen because its properties are wellknown. Thickness chosen for sample 2 implies signal overlap.

\subsection{Estimation of GA parameters}

Population size is set at 10 chromosomes, mutation rate at $20 \%$ and nonlinear parameter at 1.4 . These parameters are taken in literature for optimization procedures [23] [25] and ensure a good convergence of real-based genetic algorithms. Precision of extracted parameters depends on the number of generations. A suitable number of generations is determined through this validation. In order to test convergence of GA, very large bounds are considered for input variables (from 1000 to $8000 \mathrm{~kg} \cdot \mathrm{m}^{-3}$ for density and from 1000 to $8000 \mathrm{~m} / \mathrm{s}$ for wave velocity).

Table 3: Retrieved parameters according to number of generations, on $200 \mathrm{GA}$ iterations.

\begin{tabular}{|c|c|c|c|c|}
\hline number of generations & & 100 & 500 & 1000 \\
\hline \multirow{2}{*}{ wave velocity $(\mathrm{m} / \mathrm{s})$} & mean value $\mu$ & 6366 & 6365 & 6365 \\
\cline { 2 - 5 } & standard deviation $\sigma$ & 4.95 & 0.60 & 0.21 \\
\hline \multirow{2}{*}{ density $\mathrm{kg} / \mathrm{m}^{-3}$} & mean value $\mu$ & 2744 & 2764 & 2764 \\
\cline { 2 - 5 } & standard deviation $\sigma$ & 51.5 & 3.7 & 1.2 \\
\hline
\end{tabular}

As expected, standard deviation $\sigma$ decreases with the number of generations and all parameters converge (table 3 ). The observed precision at 500 generations is low enough to be used for following optimization.

\subsection{Results on aluminum plates}

Acoustic parameters are retrieved after comparison between experimental and theoretical spectra,normalized by the maximum amplitude of the reference spectrum. Results for both samples are shown in figure 5. Reference pulse duration corresponds to a pulse length of $8.3 \mathrm{~mm}$ in aluminum. Ratios thickness over pulse length are 0.96 and 0.17 respectively for samples 1 and 2. So spectrum of signal passing through sample 2 corresponds to an highly overlapped temporal signal. In addition number of resonances is lower in sample 2 than in sample 1 in studied bandwidth. Nevertheless, a good agreement is observed for both spectra. Frequency differences $\Delta f$ between two resonance peaks are 0.8 and $2.2 \mathrm{MHz}$ for samples 1 and 2 respectively. Using $\Delta f=c_{a l u} / 2 t h_{a l u}$ and wave velocity in aluminum $\left(c_{a l u}\right)$, retrieved thichnesses $\left(t h_{a l u}\right)$ are 4 and $1.45 \mathrm{~mm}$ respectively, as expected.
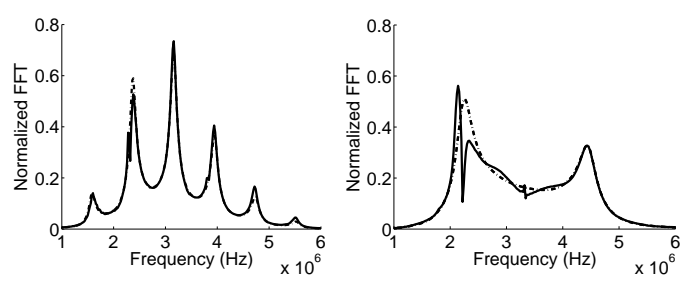

Figure 5: Comparison between experimental (solid lines) and theoretical (dashed lines) spectra: case of sample 1 (left) and sample 2 (right). 
Retrieved parameters are listed in table 4. For sample 1, these parameters are very close to theoretical ones (less than $1.2 \%$ difference).

Table 4: Solution of the GA for non overlapped (sample 1) and overlapped (sample 2) signals.

\begin{tabular}{|r|c|c|}
\hline & wave velocity $(\mathrm{m} / \mathrm{s})$ & density $\left(\mathrm{kg} / \mathrm{m}^{-3}\right)$ \\
\hline \hline sample 1 error & 6365 & 2764 \\
\hline \hline sample 2 error & $6.2 \%$ & $2.4 \%$ \\
\hline & $0.03 \%$ & 2787 \\
\hline \hline theoretical & 6380 & $3.2 \%$ \\
\hline
\end{tabular}

In the case of sample 2, relative error is less than $3.2 \%$.(table 4). Even if this relative error is higher than for sample 1, the precision of retrieved parameters is sufficient for our application.

\section{Measurements on PoSi samples}

Measurements are now performed on PoSi samples described in section 2. In this case, retrieved parameters are density, wave velocity, and thickness of porous silicon. Experimental setup is similar to the one shown in figure 2 except that transducers have a $20 \mathrm{MHz}$ center frequency. Due to wafer thickness, overlap is observed for both samples in time domain.

Theoretical transmission spectra are compared to the experimental ones in figure 6 for the case of bulk silicon (top) and porous silicon sample (bottom) and retrieved values are given in tables 5 and 6.
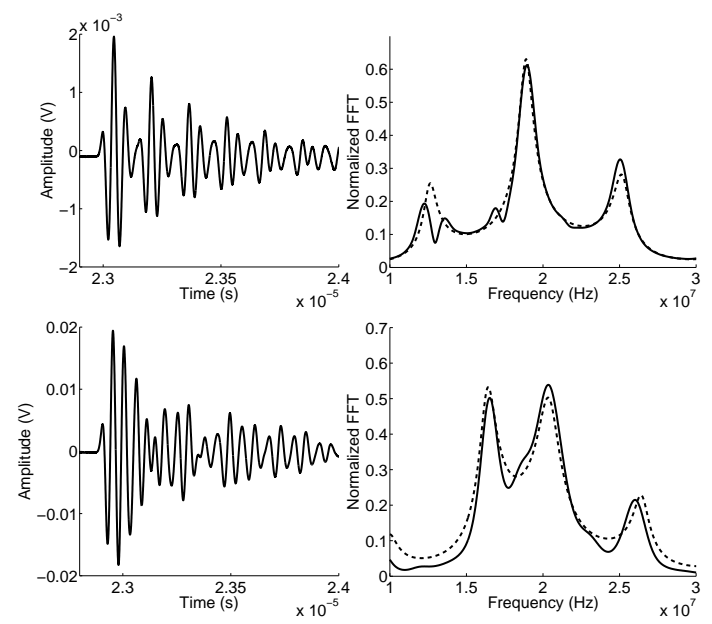

Figure 6: Time domain signal and transmission spectra through the bulk silicon wafer (top) and porous silicon wafer (bottom). Theoretical spectra obtained after optimization (dashed lines) are compared to experimental spectra (solid lines).

In the case of the porous silicon sample, a higher number of resonances can be observed because of its multilayer structure. This is an advantage for inverse problem resolution.
Table 5: Retrieved parameters of bulk silicon compared to values from literature [16].

\begin{tabular}{|c|c|c|}
\hline & density $\left(\mathrm{kg} / \mathrm{m}^{-3}\right)$ & wave velocity $(\mathrm{m} / \mathrm{s})$ \\
\hline \hline Bulk silicon & 2312 & 8500 \\
\hline literature & 2330 & 8430 \\
\hline error & $0.04 \%$ & $0.8 \%$ \\
\hline
\end{tabular}

Table 6: Retrieved parameters of porous silicon sample compared to estimations assuming $50 \%$ porosity.

\begin{tabular}{|c|c|c|c|}
\hline & density $\left(\mathrm{kg} / \mathrm{m}^{-3}\right)$ & wave velocity $(\mathrm{m} / \mathrm{s})$ & $\begin{array}{c}\text { porous layer } \\
\text { thickness }(\mu \mathrm{m})\end{array}$ \\
\hline \hline Porous silicon & 1777 & 5160 & 204 \\
\hline $\begin{array}{c}\text { estimations assuming } \\
50 \% \text { porosity }\end{array}$ & 1650 & $\begin{array}{c}5125[26] \text { at a density } \\
\text { of } 1775 \mathrm{~kg} / \mathrm{m}^{-3}\end{array}$ & $195-204$ \\
\hline error & $7.7 \%$ & $0.6 \%$ & $/$ \\
\hline
\end{tabular}

The higher error compared to the case of bulk silicon can be partly explained by an imperfect interface between porous silicon and silicon, whereas porous layer is considered as homogeneous and isotropic in the model. Yet, homogeneity hypothesis can be taken because of the high ratio between wavelength and pore size. This material is highly hydrophilic and agitation has been performed in order to avoid that air bubbles be buried in pores. Indeed, such bubbles would cause a decrease of acoustic impedance.

Retrieved data are close to expected ones (tables 5 and 6). Retrieved thickness of the porous silicon layer is in agreement with the measured one, with low error because this parameter is mainly dependent on resonance frequencies. Moreover, error on density stays between bounds dues to technological dispersion (between 5 to 10\%). Taking into account this density, retrieved wave velocity is very close to the value measured by Da Fonseca [26] for a similar material.

\section{6. conclusion}

In this work, a one dimensional model of ultrasonic wave propagation through an immersed sample was implemented and water immersion high bandwidth measurements were performed. An inverse problem resolution using a GA allowed acoustical parameters to be retrieved.

Convergence of GA using ultrasonic transmission spectra was validated on a reference material. It was verified that GA converges to final solutions that are always similar whatever the chosen initial values.

A good agreement with literature values is observed, even in the case of overlapped signals. Relative errors are similar in both cases and discrepancies can be partly explained by sample imperfections.

Further measurements will be performed in order to test sensitivity of this method on porous silicon samples with different kinds of porosity (rates, sizes, shapes). This method can also 
be used for materials in which acoustical parameters have large variations, such as polymerizing plastics or gels.

\section{References}

[1] N. Nguyen, M. Lethiecq, B. Karlsson, F. Patat, Highly attenuative rubber modified epoxy for ultrasonic transducer backing applications, Ultrasonics 34 (6) (1996) 669-675. doi:10.1016/0041-624X(96)000649.

[2] L. Wilson, Frequency domain processing for ultrasonic attenuation measurement in liver, Ultrasonic Imaging 6 (3) (1984) 278-292. doi:10.1016/0161-7346(84)90013-0.

[3] W. A. Simpson, Time-frequency-domain formulation of ultrasonic frequency analysis, The Journal of the Acoustical Society of America 56 (6) (1974) 1776. doi:10.1121/1.1903512.

[4] J. Fortineau, E. Le Clezio, F. V. Meulen, G. Feuillard, S. Barre, 3C2 Moisture Content Characterization in Composite Materials Based on Transmission Measurements, in: 2006 IEEE Ultrasonics Symposium, IEEE, 2006, pp. 232-235. doi:10.1109/ULTSYM.2006.71.

[5] V. K. Kinra, V. R. Iyer, Ultrasonic measurement of the thickness, phase velocity, density or attenuation of a thin-viscoelastic plate. Part I: the forward problem, Ultrasonics 33 (2) (1995) 95-109. doi:10.1016/0041624X(94)00025-K.

[6] T. Pialucha, P. Cawley, The detection of thin embedded layers using normal incidence ultrasound, Ultrasonics 32 (6) (1994) 431-440. doi:10.1016/0041-624X(94)90062-0.

[7] A. I. Lavrentyev, Ultrasonic spectroscopy of imperfect contact interfaces between a layer and two solids, The Journal of the Acoustical Society of America 103 (2) (1998) 657. doi:10.1121/1.423235.

[8] F. Hägglund, J. Martinsson, J. E. Carlson, C. Carlander, Model-Based Characterization of Thin Layers Using Pulse-Echo Ultrasound (2) (2007) 2-5. doi:10.3728/ICUltrasonics.2007.Vienna.1562.

[9] J. Martinsson, F. Hägglund, J. E. Carlson, Complete post-separation of overlapping ultrasonic signals by combining hard and soft modeling., Ultrasonics 48 (5) (2008) 427-443. doi:10.1016/j.ultras.2008.03.003.

[10] J. E. Carlson, F. Hägglund, Characterization of thin layers in multi-layered structures: On the problem of finding starting values for numerical solutions to inverse problems, in: 2009 IEEE International Ultrasonics Symposium, IEEE, 2009, pp. 1537-1540. doi:10.1109/ULTSYM.2009.5442036.

[11] G. Rudolph, Convergence Analysis of Canonical Genetic Algorithms, IEEE Transactions on Neural Networks 5 (1994) 96-101.

[12] M. Capelle, J. Billoué, P. Poveda, G. Gautier, A. S. Anodization, NType Porous Silicon Substrates for Integrated RF Inductors 58 (11) (2011) 4111-4114.

[13] S. OZDEMIR, J. GOLE, The potential of porous silicon gas sensors, Current Opinion in Solid State and Materials Science 11 (5-6) (2007) 92-100. doi:10.1016/j.cossms.2008.06.003.

[14] V. Lehmann, R. Stengl, A. Luigart, On the morphology and the electrochemical formation mechanism of mesoporous silicon, Materials Science and Engineering: B 69-70 (2000) 11-22. doi:10.1016/S09215107(99)00286-X.

[15] W. D. Wilson, Speed of sound in distilled water as a function of temperature and pressure 816 (1955).

[16] J. J. Wortman, R. a. Evans, Young's Modulus, Shear Modulus, and Poisson's Ratio in Silicon and Germanium, Journal of Applied Physics 36 (1) (1965) 153. doi:10.1063/1.1713863.

[17] P. Cervenka, P. Challande, A new efficient algorithm to compute the exact reflection and transmission factors for plane waves in layered absorbing media, The Journal of the Acoustical Society of America.

[18] P. He, Direct measurement of ultrasonic dispersion using a broadband transmission technique, Ultrasonics 37 (1) (1999) 67-70. doi:10.1016/S0041-624X(98)00040-7.

[19] J. H. Holland, Adaptation in Natural and Artificial Systems: An Introductory Analysis with Applications to Biology, Control and Artificial Intelligence, MIT Press, Cambridge, MA, USA, 1992.

[20] C. Z. Janikow, Z. Michalewicz, An Experimental Comparison of Binary and Floating Point Representations in Genetic Algorithms, in: ICGA, 1991, pp. 31-36.

[21] D. E. Goldberg, Genetic algorithms in search, optimization, and machine learning, Artificial Intelligence, Addison-Wesley, 1989.
[22] D. E. Goldberg, Real-coded Genetic Algorithms, Virtual Alphabets, and Blocking, Complex Systems 5 (1990) 139-167. doi:10.1.1.52.9880.

[23] Z. Michalewicz, Genetic algorithms + data structures = evolution programs (3rd ed.), Springer-Verlag, London, UK, UK, 1996.

[24] J. E. Baker, Reducing bias and inefficiency in the selection algorithm, in: Proceedings of the Second International Conference on Genetic Algorithms on Genetic algorithms and their application, L. Erlbaum Associates Inc., Hillsdale, NJ, USA, 1987, pp. 14-21.

[25] R. Haupt, Optimum population size and mutation rate for a simple real genetic algorithm that optimizes array factors, in: IEEE Antennas and Propagation Society International Symposium. Transmitting Waves of Progress to the Next Millennium. 2000 Digest. Held in conjunction with: USNC/URSI National Radio Science Meeting (Cat. No.00CH37118), Vol. 2, IEEE, pp. 1034-1037. doi:10.1109/APS.2000.875398.

[26] R. J. Da Silva Camara Mauricio Da Fonseca, J. Attal, Microcaractérisation élastique de matériaux poreux par signature acoustique $=$ Elastic microcharacterization of porous materials by acoustic signature (1995). 